\title{
A New Approach to Performance Evaluation of Generalized Selection Diversity Receivers in Wireless Channels
}

\author{
A. Annamalai ${ }^{1}$ and C. Tellambura ${ }^{2}$ \\ 1. The Bradley Department of Electrical and Computer Engineering, Virginia Tech, VA 24061 USA \\ E-mail: annamalai@vt.edu \\ 2. Faculty of Information Technology, Monash University, Clayton, Victoria 3168, Australia \\ E-mail: chintha@dgs.monash.edu.au
}

\begin{abstract}
Motivated by potential applications to wideband cellular DS-CDMA and millimeter-wave communications, study on the generalized selection combining (GSC) receiver that adaptively combines a subset of $M$ "strongest" paths out of $L$ available paths has intensified over the past few years. The study of $\operatorname{GSC}(M, L)$ receiver is also important from a theoretical standpoint because this model encapsulates both the classical selection combining and maximal-ratio combining receiver as limiting cases. Despite its importance, published results on $\operatorname{GSC}(M, L)$ receiver performance in a generalized fading channel is still very limited, mainly due to the mathematical difficulty encountered while computing the first-order statistics of a linear sum of ordered random variables. This paper provides a partial solution to the problem on hand by deriving relatively simple-to-evaluate expressions for the MGF of GSC output SNR in a variety of fading environments given that the individual branch SNRs are independent and identically distributed. Moreover, our generic single integral expression for the MGF of GSC output SNR reduces to a closed-form formula if the branch amplitudes follow either Rayleigh or Nakagami-m (positive integer fading index) distribution. An easily programmable recursive solution of the MGF in Nakagami-m channels is also provided. Our expressions hold for any $M$ and $L$ values, and thus facilitate a comprehensive analysis of GSC systems including the average symbol error probability (ASEP) analysis of a broad class of binary and $M$-ary modulations, average combined SNR and the outage rate of error probability analysis.
\end{abstract}

\section{INTRODUCTION}

The GSC diversity scheme has received considerable attention in existing literature (i.e., scanning the literature, we find that that there are more than 17 published journal and conference papers on this subject over the past four years). This is owing to its ability to mitigate the detrimental effects of deep fades on wireless channels, while achieving a good compromise between receiver performance and implementation complexity (notably fewer electronics and lower power consumption). For instance, in wideband CDMA applications, the number of available correlators will limit the number of multipaths that can be utilized in a typical rake combiner. The $\operatorname{GSC}(M, L)$ receiver clearly merits consideration because it outperforms the $M$-MRC (i.e., signal combination from only the first $M$ rake fingers) receiver configuration, owing to the improved SNR statistic at the output of the hybrid combiner. It also reduces the complexity of implementation for antenna arrays using millimeter wave communications and improves the throughput performance of packet radio networks employing "selec- tive packet combining". To explain the operation of a GSC receiver, first consider the statistically independent and identically distributed (i.i.d) random variables (RVs) $\gamma_{1}, \gamma_{2}, \ldots, \gamma_{L}$ representing the SNRs of $L$ diversity branches. If the RVs are rearranged in descending order and then written as $\gamma_{(1)} \geq \gamma_{(2)} \geq \ldots \geq \gamma_{(L)}, \gamma_{(i)}$ is called the $i$-th order statistic. We shall denote $\operatorname{GSC}(M, L)$ receiver, where $1 \leq M \leq L$, as an optimal linear combining technique applied to the subset $\gamma_{(1)}, \gamma_{(2)}, \ldots, \gamma_{(M)}$. Clearly, $\operatorname{GSC}(1, L)$ and $\operatorname{GSC}(L, L)$ are simply the well-known selection combining (SC) and classical maximal-ratio combining (MRC) receiver, respectively. Therefore, the study of the GSC receiver is important, both from a theoretical standpoint and from a practical viewpoint.

Despite this, analysis of $\operatorname{GSC}(M, L)$ receiver performance in fading environments other than Rayleigh fading is rather limited. The primary difficulty stems from the fact that the ordered SNRs $\gamma_{(i)}$, because of the inequalities among them, are necessarily dependent. Consequently, finding the moment generating function (MGF) of a linear sum of ordered RVs $\gamma=\sum_{i=1}^{M} \gamma_{(i)}$ (i.e., GSC output SNR) is generally much more difficult than for the unordered $R V s^{1}$. In the past [4]-[8], numerous ad-hoc attempts have been made to compute the MGF of ordered Gamma variates (i.e., Nakagami-m fading case), resulting in various complicated formulas. Furthermore, the existing mathematical approaches do not lend themselves to the performance evaluation of GSC receivers in Rician and Nakagami-q channels. As such, one of the main objectives of this paper is to report a novel mathematical framework which can facilitate the problem of analyzing the GSC receiver performance with i.i.d diversity branches in a variety of fading environments. An attractive feature of our approach is that the MGF of $\operatorname{GSC}(M, L)$ output SNR for all common fading channel models as well as for all combinations of $M$ and $L$ values can be simply expressed in terms of only a single integral whose integrand is composed of tabulated functions. For the special cases of Rayleigh and Nakagami-m fading with positive integer fading severity index, the integral simplifies into a closed-form formula.

1. For exponential RVs (i.e., Rayleigh fading case), however, the $\gamma_{(i)}$ may be simply expressed as a linear combination of $i$ unordered standard exponential RVs with an appropriate weighting [1]. 
The significance of this study also lies in that many of the previously reported $\operatorname{GSC}(M, L)$ results over Nakagami-m fading channels have a number of limitations. In [2], the final result for the error probability involves the computation of multiple integrals (undesirable for larger values of $M$ ). The main limitation of [3] is that the GSC results are only applicable to $M=2$ and $L=3$ or 4 . Extending this approach to larger $M$ values appears to be impossible [4]. Apart from coherent BPSK and BFSK, [3] did not analyze other higher level modulation formats such as MPSK, M-ary square QAM, star-QAM, DQPSK and MDPSK, to name a few. In [4], the authors applied the Dirichlet transformation to simplify a multiple integral for the MGF of GSC output SNR. The resulting expression for the MGF [4, Eq. (15)] generally requires the evaluation of $(L-1)$-fold nested integral via numerical methods, which can be tedious and complicated, particularly for large $L$ even if $M$ is small (e.g., $M=1$ ). Also, for the positive integer $m$ case, one has to simplify the $(L-1)$-fold integral for the MGF for each different $M$ and/or $L$ values! As such, the approach does not lend itself to a versatile method for calculating the ASEP for arbitrary $M$ and $L$. More recently, [5] presents ASEP analysis of coherent and noncoherent $\operatorname{GSC}(M, L)$ receivers in Nakagami- $m$ fading channels for both the i.i.d and the nonidentical fading statistics cases using an MGF approach. An equivalent MGF formula for the i.i.d Nakagami-m fading case was discovered independently in [6]-[7], in addition to the derivation of the average combined SNR and outage probability analysis of GSC systems. In [8], we refined the analysis presented in [7] and derived the ASEP of a multitude of digital modulation schemes in closed-form.

In this paper, by contrast with above, we first derive a considerably simpler expression for the MGF of SNR at the output of an hybrid $\operatorname{GSC}(M, L)$ combiner. Besides, our formula is not restricted to the Nakagami-m channels alone but rather applies to any other fading channel models such as the Rician fading. Different from [4], our initial formulation of the above ordered statistics problem will require evaluation of an $M$-fold integral for the MGF and the resulting expression holds for arbitrary $L$. Using an appropriate variable substitution, we were able to simply the $M$-fold nested integral into a single integral whose integrand is composed of only the PDF of a single diversity branch and its marginal MGF (see Appendix A). Further simplifications of this integral are also possible for several special cases. For instance, the integral can be evaluated in closed-form when the Nakagami-m fading severity index, $m$, assumes a positive integer value. For the real $m$ case, the integral may be replaced by a single infinite series for $M \leq 2$ or a two-fold infinite sum for any $M \geq 3$. These MGFs can then be used to compute various performance metrics of a coherent GSC receiver: (i) ASEP of a variety of binary and multilevel modulation schemes; (ii) outage rate of error probability; (iii) average combined SNR.
Besides, the MGF expression derived in this paper also applies to the performance evaluation of MFSK and DPSK in conjunction with a noncoherent GSC (post-diztection $\mathrm{SC} / \mathrm{EGC}$ ) quadratic receiver over wireless channels.

II. $\operatorname{GSC}(M, L)$ COMBINER OUPUT STATISTIC

In this section, we derive analytical expressions for the $\operatorname{GSC}(M, L)$ combiner output statistics by modelling the branch amplitudes as i.i.d Rayleigh, Rician, Nakagami-m or Nakagami-q RVs. These expressions can be applied directly for computing the ASEP and outage probability for different modulation schemes.

From [9], we know that when $M$ strongest diversity branches are selecteci from a total of $L$ available i.i.d diversity branches the joint PDF is given by

$$
p_{\gamma_{1}, \ldots, \gamma_{M}}\left(\gamma_{1}, \ldots, \gamma_{M}\right)=M !\left(\begin{array}{c}
L \\
M
\end{array}\right)\left[F\left(\gamma_{M}\right)\right]^{L-M} \prod_{i=1}^{M} p\left(\gamma_{1}\right)
$$

where $\gamma_{1} \geq \ldots \geq \gamma_{M} \geq 0, p($.$) and F($.$) correspond to the PDF$ and CDF, respectively, of the SNR for a single channel reception (no-diversity case). Recognizing that the MGF of the combiner's output SNR $\phi_{\gamma}($.$) is the key to the unified$ analysis of a wide range of modulation schemes over wireless channels, our immediate intention will be to derive the desired MGF.

Let $\gamma=\sum_{i=1}^{M} \gamma_{(i)}$ denote the hybrid combiner output SNR. Then, the MGF of $\gamma$ may be computed as [8]

$$
\begin{aligned}
\phi_{Y}(s)= & \int_{0}^{\infty} e^{-s \gamma_{1}} \int_{0}^{\gamma_{1}} e^{-s \gamma_{2}} \ldots \int_{0}^{\gamma_{M-1}} e^{-s \gamma_{M}} p_{\gamma_{1}} \ldots \gamma_{M}\left(\gamma_{1}, \ldots, \gamma_{M}\right) d \gamma_{M} \ldots d \gamma_{2} d \gamma_{1} \\
= & M !\left(\begin{array}{l}
L \\
M
\end{array}\right) \int_{0}^{\infty} e^{-s \gamma_{1}} p\left(\gamma_{1}\right) \int_{0}^{\gamma_{1}} e^{-s \gamma_{2}} p\left(\gamma_{2}\right) \ldots \int_{0}^{\gamma_{M-2}} e^{-s \gamma_{M-1}} p\left(\gamma_{M-1}\right) \\
& \times \int_{0}^{\gamma_{M-1}} e^{-s \gamma_{M}} p\left(\gamma_{M}\right)\left[F\left(\gamma_{M}\right)\right]^{L-M} d \gamma_{M} \ldots d \gamma_{2} d \gamma_{1} \\
= & M !\left(\begin{array}{l}
L \\
M
\end{array}\right) \int_{0}^{\infty} e^{-s \gamma_{M}} p\left(\gamma_{M}\right)\left[F\left(\gamma_{M}\right)\right]^{L-M} \\
& \times \int_{\gamma_{M}} e^{-s \gamma_{M-1}} p\left(\gamma_{M-1}\right) \ldots \int_{\gamma_{2}} e^{-s \gamma_{1}} p\left(\gamma_{1}\right) d \gamma_{1} \ldots d \gamma_{M-1} d \gamma_{M}
\end{aligned}
$$

From Appendix A, we know that (2) can be re-written very concisely as

$$
\phi_{\gamma}(s)=M\left(\begin{array}{l}
L \\
M
\end{array}\right) \int_{0} e^{-s \gamma_{M}} p\left(\gamma_{M}\right)\left[F\left(\gamma_{M}\right)\right]^{L-M}\left[\phi\left(s, \gamma_{M}\right)\right]^{M-1} d \gamma_{M}
$$

where $\phi(s, y)=\int_{y} e^{-s x} p(x) d x$ is the marginal MGE of SNR of a single diversity branch (i.e., no-diversity). Fortunately, closed-form solutions for $\phi(s, y)$ are available for all the statistical channel models that are typically used to characterize the variations in the received signal power over wireless (fading) channels. They are summarized below:

A. Rayleigh Fading

$$
\begin{aligned}
& p(x)=\frac{1}{\Omega} \exp (-x / \Omega), x \geq 0 \\
& \phi(s, y)=\frac{\exp [-y(s+1 / \Omega)]}{1+s \Omega}
\end{aligned}
$$

where $\Omega:=E[x]$ denotes the average SNR per branch. B. Rician Fading

$$
p(x)=\frac{1+K}{\Omega} \exp \left[-K-\frac{(1+K) x}{\Omega}\right] I_{0}\left[2 \sqrt{\frac{K(K+1) x}{\Omega}}\right], x \geq 0
$$




$$
\begin{gathered}
\phi(s, y)=\frac{1+K}{s \Omega+K+1} Q\left(\sqrt{\frac{2 K(K+1)}{s \Omega+K+1}}, \sqrt{\frac{2(s \Omega+K+1) y}{\Omega}}\right) \\
\times \exp \left[\frac{-s K \Omega}{s \Omega+K+1}\right]
\end{gathered}
$$

where $Q(\sqrt{2 a}, \sqrt{2 b})=\int_{b} \exp (-t-a) I_{0}(2 \sqrt{a t}) d t$ is the first order Marcum Q-function, $I_{0}($.$) is the modified Bessel func-$ tion and $K \geq 0$ is the Rice factor.

C. Nakagami-m Fading

$$
\begin{gathered}
p(x)=\frac{1}{\Gamma(m)}\left(\frac{m}{\Omega}\right)^{m} x^{m-1} \exp \left(\frac{-m x}{\Omega}\right), x \geq 0 \\
\phi(s, y)=\frac{1}{\Gamma(m)}\left(\frac{m}{m+s \Omega}\right)^{m} \Gamma[m, y(s+(m / \Omega))]
\end{gathered}
$$

where $\Gamma(a, x)=\int_{x} \exp (-t) t^{-1} d t$ denotes the complementary incomplete Gamma function and $m \geq 0.5$ is the fading severity index. If the fading index $m$ assumes a positive integer value, (9) may be simplified as

$$
\phi(s, y)=\left(\frac{m}{m+s \Omega}\right)^{m} \exp \left(-s y-\frac{m y}{\Omega}\right)^{m-1} \sum_{k=0} \frac{[y(s+m / \Omega)]^{k}}{k !}
$$

\section{Nakagami-q Fading}

$$
\begin{array}{r}
p(x)=\frac{1}{\Omega \sqrt{1-b^{2}}} \exp \left[\frac{-x}{\left(1-b^{2}\right) \Omega}\right] I_{0}\left[\frac{b x}{\left(1-b^{2}\right) \Omega}\right], x \geq 0 \\
\phi(s, y)=\frac{1}{\sqrt{[s \Omega+1]^{2}-[s \Omega b]^{2}}}-\frac{1}{s\left(1-b^{2}\right) \Omega+1} \\
\times I_{e}\left(\frac{b}{s\left[1-b^{2}\right] \Omega+1}, \frac{y\left[1-b^{2}\right] \Omega}{s\left[1-b^{2}\right] \Omega+1}\right)
\end{array}
$$

where $-1 \leq b=\left(1-q^{2}\right) /\left(1+q^{2}\right) \leq 1,0 \leq q_{k} \leq \infty$ is the fading parameter and Rice's $I_{e}$-function is related to the first-order Marcum Q-function as

$$
I_{e}(V / U, U)=\frac{U}{W}[Q(\sqrt{U+W}, \sqrt{U-W})-Q(\sqrt{U-W}, \sqrt{U+W})]
$$

while $W=\sqrt{U^{2}-V^{2}}$.

It is also interesting to note that (3) can also be re-stated as

$$
\begin{aligned}
\phi_{\gamma}(s)= & M\left(\begin{array}{l}
L \\
M
\end{array}\right) \int_{0}^{r / 2} e^{-s \tan \theta} p(\tan \theta)[1-\phi(0, \tan \theta)]^{L-M} \\
& \times[\phi(s, \tan \theta)]^{M-1} \sec ^{2} \theta d \theta
\end{aligned}
$$

since $F(x)=1-\phi(0, x)$. Besides, (3) and/or (13) can be evaluated very efficiently using a Gauss-Chebychev quadrature (GCQ) approximation formula. For the specific case of i.i.d Nakagami-m channels, it is apparent that (3) or (13) will yield significant improvement over [5] in terms of computational complexity because the latter involves an $M$-dimensional GCQ sum whereas in our case, we need to compute only a one-dimensional GCQ sum!

The CDF of GSC output SNR can be readily computed by invoking Gil-Pelaez inversion theorem (which gives a relation between the CDF and MGF of a random variable) and then use a trapezoidal rule approximation, we arrive at

$$
F_{\gamma}(x) \cong \frac{1}{2}-\frac{2}{\pi} \sum_{\substack{n=1 \\ n \text { odd }}}^{\infty} \frac{1}{n} \operatorname{Imag}\left[\phi_{\gamma}\left(\frac{-j 2 \pi n}{T}\right) \exp \left(\frac{-j 2 \pi n x}{T}\right)\right]
$$

where the coefficient $T$ selected is sufficiently large such that $\operatorname{Pr}(x>T) \leq \varepsilon$, and $\varepsilon$ can be set to a very small value. Our numerical computation based on (14) indicates that this series converge slowly at low values of $F_{\gamma}(x)$ (say, $\left.F_{Y}(x)<10^{-4}\right)$. Therefore, we also provide an alternative Laplace inversion formula for calculating the CDF of GSC output SNR:

$$
F_{Y}(x)=\frac{1}{\pi} \int_{0}^{\infty} \operatorname{Real}\left\{\frac{\phi_{\gamma}(c+j t)}{c+j t} \exp [(c+j t) x]\right\} d t
$$

The optimal choice of $c$ is the saddle point that minimizes $\psi(s)=\phi_{y}(s) e^{s x} / s$ for $s>0$. Since $\psi(s)$ is a convex function in the range $[0, \infty)$, this minimum exists and unique. However, this value need not be determined with great precision. Standard mathematical software such as Matlab and Maple can readily solve for $c$. The uniqueness of the global optimal $c$ simplifies this search enormously. An absolute precision of $10^{-1}$ is sufficient for this optimization (i.e., very coarse search). Having obtained the optimal $c$, one can compute $F_{\gamma}(x)$ as accurately as one desires by using a trapezoidal sum utilizing sufficiently many samples of integrand spaced closely enough together.

We have validated that (15) is stable for numerical computation even as low as $10^{-12}$. The above development is also important because, to the best of our knowledge, there are no published results on the CDF of the GSC output SNR available in existing literature for wireless channels other than Rayleigh fading. Yet, it is useful for predicting the outage probability improvement that can be realized by using a GSC diversity receiver.

It is now easy to show that the PDF of the GSC output SNR in a Nakagami-m fading channel is given by

$$
p_{\gamma}(x)=\frac{\mathrm{d}}{\mathrm{d} x} F_{\gamma}(x) \cong \frac{4}{T} \sum_{\substack{n=1 \\ n \text { odd }}}^{\infty} \operatorname{Real}\left[\phi_{\gamma}\left(\frac{-j 2 \pi n}{T}\right) \exp \left(\frac{-j 2 \pi n x}{T}\right)\right]
$$

It is also possible to derive a closed-form formula for $p_{\gamma}(x)$ and $F_{\gamma}(x)$ in Nakagami-m (positive integer fading index) channels. They are omitted here for brevity. At this juncture, it is also worth noting that $\phi_{\gamma}(s)$ can be evaluated using a recursion formula for Nakagami-m channels (for both positive integer and real $m$ values) directly from (2):

(a) positive integer $m$

$$
\begin{aligned}
\phi_{\gamma}(s)= & \frac{M !}{[\Gamma(m)]^{M}}\left(\begin{array}{l}
L \\
M
\end{array}\right) \sum_{k=0}^{L-M}(-1)^{k}\left(\begin{array}{c}
L-M \\
k
\end{array}\right) \sum_{z=0}^{k(m-1)} \beta(z, k, m)\left(\frac{m}{\bar{\gamma}}\right)^{m M+z} \\
& \times F\left(s+\frac{m}{\bar{\gamma}}, s+\frac{m}{\bar{\gamma}}(1+k), m, z+m, M\right)
\end{aligned}
$$

where $F(a, b, m, n, 1)=\Gamma(n) / b^{n}$,

$$
\begin{gathered}
F(a, b, m, n, M)=\frac{(n-1) !}{b^{n}}\left\{\frac{a^{m(1-M)}}{(M-1) !}[(m-1) !]^{M-1}-\sum_{k=0}^{n-1} \frac{b^{k}}{k !}\right. \\
\times F(a, a+b, m, k+m, M-1)\}, M \geq 2
\end{gathered}
$$

and the coefficients $\beta(., \ldots)$ may be computed using multinomial theorem, 


$$
\beta(z, k, d)=\sum_{i=z-d+1}^{z} \frac{\beta(i, k-1, d)}{(z-i) !} I_{(0,(k-1)(d-1)\}}(i)
$$

while $I_{[a, b]}(i)=\left\{\begin{array}{ll}1 & a \leq i \leq b \\ 0 & \text { otherwise }\end{array}, \quad \beta(0,0, d)=\beta(0, k, d)=1\right.$,

$\beta(z, 1, d)=1 / z$ ! and $\beta(1, k, d)=k$.

(b) real $m$

$$
\begin{gathered}
\phi_{\gamma}(s)=\frac{M !}{[\Gamma(m)]^{M}}\left(\begin{array}{l}
L \\
M
\end{array}\right) \sum_{n=0}^{\infty} c_{n}\left(\frac{m}{\bar{\gamma}}\right)^{n+m L} \\
\times G\left(s+\frac{m}{\bar{\gamma}}, s+\frac{m}{\bar{\gamma}}(L-M+1), m, n+m(L-M+1), M\right)
\end{gathered}
$$

where

$$
G(a, b, m, v, M)=\sum_{k=0}^{\infty} \frac{b^{k} G(a, a+b, m, k+v+m, M-1)}{\Gamma(1+v+k) / \Gamma(v)}
$$

Readers are referred to [8] for a detailed description on the applications of the PDF, CDF and the MGF formulas in the characterization of $\operatorname{GSC}(M, L)$ diversity systems.

\section{COMPUTATIONAL RESULTS}

Fig. 1 and Fig. 2 illustrate the effects of combining additional ordered SNRs on the ASEP performance of QPSK in Nakagami-m fading channels. While the probability of deep fades decreases as the number of combined branches $M$ increases, the marginal value of higher order diversities diminishes, however. For the same reason, the amount of diversity improvement diminishes as the channel condition improves (compare Fig. 1 and Fig. 2). It is also evident from these figures that increasing $L$ also translates into a considerable improvement in the receiver performance.
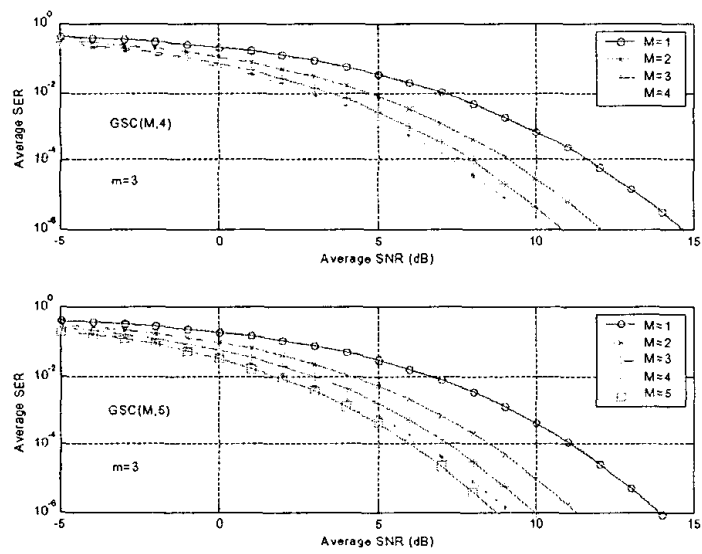

Fig. 1. ASEP of QPSK versus the mean SNR/symbol/branch $\Omega$ for $m=3$ and $L=4$ or $L=5$.

Using Fig. 3, it is possible to predict the outage probability of a specified modulation scheme that employs a $\operatorname{GSC}(M, 5)$ receiver in Nakagami-m channels ( $m=2$ ) when the average $\mathrm{SNR} /$ symbol/branch is given, or alternatively, compute the mean SNR/symbol/branch to satisfy an outage requirement. For instance, if $P_{e} *=10^{-3}$ is specified, then $P_{\text {out }}=F_{\gamma}(4.77)$ for BPSK because the threshold SNR may be computed as $\Omega^{*}=\left[\mathrm{erfc}^{-1}\left(2 \times 10^{-3}\right)\right]^{2}=4.77$. Alternatively, if $P_{\text {out }}=10^{-2}$ is specified, then the mean SNR/branch requirement may be estimated as $\Omega=4.77(0.6)=4.6 \mathrm{~dB}$ (interpolated from Fig. 3 ) assuming that $m=2, M=3$ and $L=5$. If 4-QAM (or QPSK) is used rather than BPSK, then the mean SNR/branch requirement increases to $\Omega=10.83(0.6)=8.1 \mathrm{~dB}$.
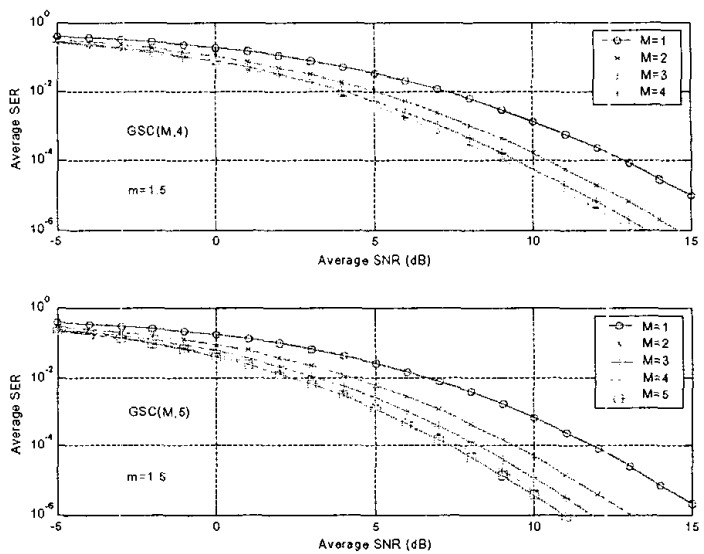

Fig. 2. ASEP of QPSK versus the mean SNR/symbol/brinch $\Omega$ for $m=1.5$ and $L=4$ or $L=5$.

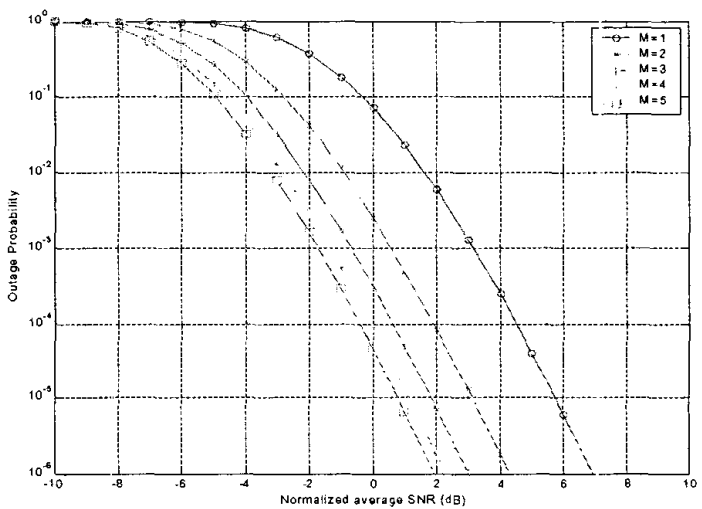

Fig. 3. Outage probability $F_{\gamma}\left(\Omega^{*}\right)$ versus the normalized average $\mathrm{SNR} /$ symbol/branch $\Omega / \Omega^{*}$ for $\operatorname{GSC}(M, 5)$ receiver on a Nakagami-m channel $(m=2)$

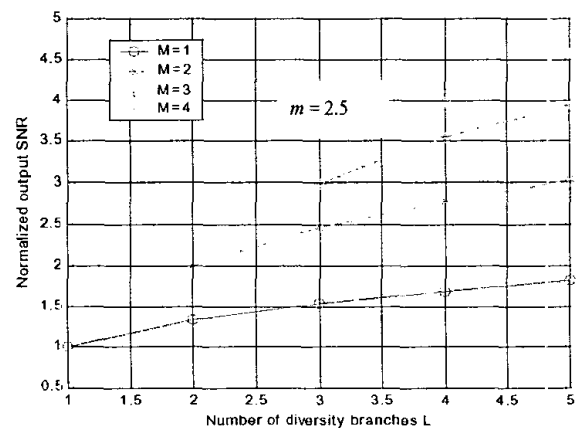

Fig. 4. The normalized average GSC output SNR $\vec{\gamma}_{s s c} / \Omega 2$ versus the total number of diversity branches $L$ for various $M$ in a Nakagami-m channel. 
In Fig. 4, the normalized mean combined SNR at the GSC output is plotted as a function of diversity order $L$ for different $M$ values. For a fixed $M$, we observe that $\bar{\gamma}_{g s c} / \Omega$ increases with increasing $L$. However, the relative increase declines gradually as $(L-M)$ increases, which is typical of selection diversity systems. Also, for a fixed $L$, increasing $M$ leads to a higher $\bar{\gamma}_{g s c} / \Omega$ as anticipated. APPENDIX A

If the i.i.d random variables $\gamma_{1}, \gamma_{2}, \ldots, \gamma_{L}$, each with PDF $p(x)$ and CDF $F(x)$, are arranged in a descending order of magnitude and then written as $\gamma_{1: L} \geq \gamma_{2: L} \geq \ldots \geq \gamma_{L: L}$, we call $\gamma_{k: L} \equiv \gamma_{(k)}$ the $k$-th order statistic. Recognizing that the knowledge of MGF of $\gamma=\sum_{k=1}^{M} \gamma_{k: L}$ can be used to unify the performance analysis of digital communication systems over fading channels, in this appendix, we will develop a general procedure for deriving $\phi_{\gamma}(s)$ for any $1 \leq M \leq L$ and also without imposing any restrictions on the fade distribution. From (2), we have

$$
\begin{gathered}
\phi_{\gamma}(s)=M !\left(\begin{array}{l}
L \\
M
\end{array}\right) \int_{0}^{\infty} e^{-s \gamma_{M}} p\left(\gamma_{M}\right)\left[F\left(\gamma_{M}\right)\right]^{L-M} \\
\times \int_{\gamma_{M}}^{\infty} e^{-\gamma \gamma_{M-1}} p\left(\gamma_{M-1}\right) \ldots \int_{\gamma_{2}} e^{-s \gamma_{1}} p\left(\gamma_{1}\right) d \gamma_{1} \ldots d \gamma_{M-1} d \gamma_{M}
\end{gathered}
$$

Now let us consider several special cases. For $M=1$ (SC), (A.1) reduces to

$$
\phi_{\mathrm{\gamma}}(s)=L \int_{0}^{\infty} e^{-s \gamma_{1}} p\left(\gamma_{1}\right)\left[F\left(\gamma_{1}\right)\right]^{L-1} d \gamma_{1}
$$

For $M=2,($ A.1) simplifies into

$$
\begin{aligned}
\phi_{\gamma}(s) & =2\left(\begin{array}{l}
L \\
2
\end{array}\right) \int_{0} e^{-s \gamma_{2}} p\left(\gamma_{2}\right)\left[F\left(\gamma_{2}\right)\right]^{L-2}\left\{\int_{\gamma_{2}} e^{-s \gamma_{1}} p\left(\gamma_{1}\right) d \gamma_{1}\right\} d \gamma_{2} \\
& =2\left(\begin{array}{l}
L \\
2
\end{array}\right) \int_{0}^{\infty} e^{-s \gamma_{2}} p\left(\gamma_{2}\right)\left[F\left(\gamma_{2}\right)\right]^{L-2} \phi\left(s, \gamma_{2}\right) d \gamma_{2}
\end{aligned}
$$

where $\phi(s, y)=\int_{y} e^{-s x} p(x) d x$ is the marginal MGF of RV $\gamma_{k}$ and $k \in\{1,2, \ldots, L\}$.

Letting $M=3$ in (A.1), we obtain

$$
\begin{gathered}
\phi_{\gamma}(s)=6\left(\begin{array}{l}
L \\
3
\end{array}\right) \int_{0} e^{-s \gamma_{3}} p\left(\gamma_{3}\right)\left[F\left(\gamma_{3}\right)\right]^{L-3} \\
\times\left\{\int_{\gamma_{3}} e^{-s \gamma_{2}} p\left(\gamma_{2}\right) \phi\left(s, \gamma_{2}\right) d \gamma_{2}\right\} d \gamma_{3}
\end{gathered}
$$

Using variable substitution $u=\phi\left(s, \gamma_{2}\right)$ in the inner integral of (A.4), and after simplifications, we obtain

$$
\phi_{\gamma}(s)=3\left(\begin{array}{l}
L \\
3
\end{array}\right) \int_{0}^{\infty} e^{-s \gamma_{3}} p\left(\gamma_{3}\right)\left[F\left(\gamma_{3}\right)\right]^{L-3}\left[\phi\left(s, \gamma_{3}\right)\right]^{2} d \gamma_{3}
$$

with the aid of the integral identity

$$
\int_{a}^{b}[g(x)]^{n} g^{\prime}(x) d x=\frac{[g(b)]^{n+1}-[g(a)]^{n+1}}{n+1}
$$

and recognizing that $d u=-e^{-s \gamma_{2}} p\left(\gamma_{2}\right) d \gamma_{2}$.

Using the procedure described above, it is straight-forward to show that for $M=4$, (A.1) reduces to

$$
\phi_{\gamma}(s)=4\left(\begin{array}{l}
L \\
4
\end{array}\right) \int_{0}^{\infty} e^{-s \gamma_{4}} p\left(\gamma_{4}\right)\left[F\left(\gamma_{4}\right)\right]^{L-4}\left[\phi\left(s, \gamma_{4}\right)\right]^{3} d \gamma_{4}
$$

In summary, we can show that the $(M-1)$-fold nested integral in (A.1) may be replaced by a product of $(M-1)$ integrals by applying (A.6) repetitively, viz.,

$$
\int_{\gamma_{M}} e^{-s \gamma_{M-1}} p\left(\gamma_{M-1}\right) \ldots \int_{\gamma_{2}}^{m} e^{-s \gamma_{1}} p\left(\gamma_{1}\right) d \gamma_{1} \ldots d \gamma_{M-1}=\frac{\left[\phi\left(s, \gamma_{M}\right)\right]^{M-1}}{(M-1) !}(A
$$

Substituting (A.8) into (A.1), we obtain an elegant formula for the MGF of GSC output SNR:

$$
\phi_{\gamma}(s)=M\left(\begin{array}{l}
L \\
M
\end{array}\right) \int_{0}^{e} e^{-s \gamma_{M}} p\left(\gamma_{M}\right)\left[F\left(\gamma_{M}\right)\right]^{L-M}\left[\phi\left(s, \gamma_{M}\right)\right]^{M-1} d \gamma_{M}
$$

which is valid for all combinations of $L$ and $M \leq L$ as well as for different fading environments.

At this juncture, it is instructive to examine $\phi_{y}($.$) for the two$ limiting cases of interest. When $M=1$, (A.9) is in agreement with (A.2). On the other extreme (i.e., $M=L$ ), we get (using (A.6))

$$
\phi_{\gamma}(s)=L \int_{0}^{\infty} e^{-s \gamma_{L}} p\left(\gamma_{L}\right)\left[\phi\left(s, \gamma_{L}\right)\right]^{L-1} d \gamma_{L}=[\phi(s)]^{L}
$$

as expected (note: $\phi(s, 0)=\phi(s)$ corresponds to the MGF of SNR in the no-diversity case).

It is also not very difficult to demonstrate that (A.9) collapses into a single integral expression with finite integration limits (see (13)) while the fading signal amplitudes follow either the Rician, or the Nakagami-m (real $m \geq 0.5$ ), or the Nakagami-q distribution. This is attributed to the availability of closed-form solutions for the marginal MGF $\phi\left(s, \gamma_{k}\right)$ in the above cases. Moreover, if $\gamma_{1}, \gamma_{2}, \ldots, \gamma_{L}$ are i.i.d exponential or Gamma variates, (A.9) can also be evaluated in closed-form by mimicking our development in [8].

\section{REFERENCES}

[1] B. Arnold, N. Balakrishnan and H. N. Nagaraja, A First Course in Order Statistics, Wiley, New York: 1992.

[2] R. Wong, A. Annamalai and V. K. Bhargava, "Evaluation of Predetection Diversity Techniques for Rake Receivers," IEEE PACRIM'97, pp. 227-230, Aug. 1997.

[3] M. Alouini and M. Simon, "Performance of Coherent Receivers with Hybrid SC/MRC over Nakagami-m Fading Channels," IEEE Transactions on Vehicular Technology, Vol. 48, pp. 1155-1164, July 1999.

[4] M. Alouini and M. Simon, "Application of the Dirichlet Transformation to the Performance Evaluation of Generalized Selection Combining over Nakagami-m Fading Channels," Journal of Communications and Networks, Vol. 1, pp. 5-13, Mar. 1999.

[5] Y. Ma and C. Chai, "Unified Error Probability Analysis for Generalized Selection Combining in Nakagami Fading Channels," IEEE Journal on Selected Areas in Communications, Vol. 18, pp. 2198-2210, Nov. 2000.

[6] C. Tellambura and A. Annamalai, Tutorial Notes: "Analytical Tools for Wireless Communications Systems Design", IEEE $V T C ' 2000$ (May 2000) and IEEE ICC'2000 (June 2000).

[7] Annamalai and Tellambura, "Error Rates of Hybrid SC/MRC Diversity Systems on Nakagami-m Channels," Proc. 2000 IEEE WCNC, pp. 227-231, Sept. 2000.

[8] A. Annamalai and C. Tellambura, "A Direct Approach to Performance Evaluation of Generalized Selection Diversity Systems over Nakagami-m Fading Channels," submitted for journal publication.

[9] A. Papoulis, Probability, Random Variables and Stochastic Processes, McGraw-Hill, New York: 1991. 